\title{
Variability of the $\mathrm{H}_{2} \mathrm{O}$ maser associated with the M-supergiant S Persei ${ }^{\star}$
}

\author{
E. E. Lekht ${ }^{1,2}$, G. M. Rudnitskij ${ }^{2}$, J. E. Mendoza-Torres ${ }^{1}$, and A. M. Tolmachev ${ }^{3}$ \\ ${ }^{1}$ Instituto Nacional de Astrofísica, Óptica y Electrónica, Luis Enrique Erro No. 1, Apdo Postal 51 y 216, 72840 Tonantzintla, \\ Puebla, México \\ e-mail: [lekht; mend] @inaoep.mx \\ 2 Sternberg Astronomical Institute, 13 Universitetskij prospekt, Moscow, 119992 Russia \\ e-mail: gmr@sai.msu.ru \\ 3 Pushchino Radio Astronomy Observatory, Astrospace Center of the Lebedev Institute of Physics, \\ Russian Academy of Sciences, Pushchino, Moscow Region, 142290 Russia \\ e-mail: tolm@prao.psn.ru
}

Received 3 January 2005 / Accepted 3 February 2005

\begin{abstract}
We present the results from observing the circumstellar maser emission of the M-type supergiant S Per in the $6_{16}-$ $5_{23}$ water-vapour line at $1.35 \mathrm{~cm}$. The observations were carried out in 1981-2002 (JD = 2444 900-2 452 480) on the RT-22 radio telescope of the Pushchino Radio Astronomy Observatory, Astrospace Center of the Lebedev Institute of Physics, Russian Academy of Sciences. The $\mathrm{H}_{2} \mathrm{O}$ spectra obtained represent an unprecedented long, uniform dataset on this star. We discuss the properties of the optical and maser variations of S Per, together with particulars of the available VLBI maps. The close relation between maser and optical variations favors a model in which mass-loss is episodic. Changes observed in the total $\mathrm{H}_{2} \mathrm{O}$ line flux follow the visual light curve with a delay of 0.01 to $0.5 P$, where $P \approx 800 \mathrm{~d}$ is the mean light cycle for S Per. The feature at $V_{\mathrm{LSR}}=-44 \mathrm{~km} \mathrm{~s}^{-1}$ flared in July 1988 , which seemed to be the response of the maser to an unusually bright optical maximum. The position of the $-44-\mathrm{km} \mathrm{s}^{-1}$ feature on the VLBI maps coincides with the direction toward the optical stellar disc, which can be explained by amplification of enhanced stellar continuum by the $\mathrm{H}_{2} \mathrm{O}$ line.
\end{abstract}

Key words. stars: AGB and post-AGB - circumstellar matter - stars: mass-loss - stars: supergiants - masers - stars: late-type

\section{Introduction}

Among several hundred late-type stars known to display circumstellar maser emission in the $\lambda=1.35 \mathrm{~cm} \mathrm{H}_{2} \mathrm{O}$ line (Benson et al. 1990), there is a relatively small group of M-type supergiants (NML Cyg, VY CMa, VX Sgr, S Per, and some others). All of them are classified as variable stars with slow optical variations, either long-period or semiregular. Their variability differs from that of late-type giants by considerably longer periods (or timescales) of light variations. The $\mathrm{H}_{2} \mathrm{O}$ maser emission of the supergiants is also variable, while the flux density changes may exceed two orders of magnitude.

From the viewpoint of long-term observations, it is of interest to compare stellar variability in the $\mathrm{H}_{2} \mathrm{O}$ line, optical and infrared ranges. Correlation between the variability curves in the $\mathrm{H}_{2} \mathrm{O}$ line and infrared, as well as accompanying changes in the line profile structure, would characterize the processes taking place in the inner layers of circumstellar envelopes of late-type supergiants. Observations in the $\mathrm{H}_{2} \mathrm{O}$ line offer a unique opportunity to study the region of outward acceleration of matter lost

* Figure 1 is only available in electonic form at: http://www. edpsciences.org by the star. The radius of the $\mathrm{H}_{2} \mathrm{O}$ maser zone approximately coincides with $R_{\mathrm{c}}$, the radius of dust condensation in circumstellar envelopes of late-type supergiants, while the main acceleration of gas starts precisely at $R_{\mathrm{c}}$ (Richards \& Yates 1998).

In addition, the particular structure of the Doppler line profile is very helpful for investigating the variability of the circumstellar $\mathrm{H}_{2} \mathrm{O}$ maser emission. In supergiant stars this structure is by far richer and more diverse than in giants. In supergiants the $\mathrm{H}_{2} \mathrm{O}$ line profile may contain up to a dozen emission peaks, spread in a radial-velocity interval $\Delta V_{\mathrm{LSR}}$ of $30 \mathrm{~km} \mathrm{~s}^{-1}$ or broader. In the $\mathrm{H}_{2} \mathrm{O}$ line profile of giants, in contrast, usually only one or two features are observable, and $\Delta V_{\mathrm{LSR}}$, as a rule, does not exceed $5 \mathrm{~km} \mathrm{~s}^{-1}$.

In this work we report $\mathrm{H}_{2} \mathrm{O}$ line observations of the M-type supergiant S Per (BD $+57^{\circ} 552$, HD 14528, IRC+60088, IRAS $02192+5821)$ in the $\mathrm{H}_{2} \mathrm{O} 6_{16}-5_{23}$ line $(\lambda=1.35 \mathrm{~cm})$, carried out from 1981 to 2002. S Per is one of the stellar objects we have been observing in the $\mathrm{H}_{2} \mathrm{O} 1.35-\mathrm{cm}$ maser line since the early 1980s (Berulis et al. 1983; Esipov et al. 1999). In our previous papers we traced in detail regularities in the $\mathrm{H}_{2} \mathrm{O}$ maser variations for several late-type stars: RR Aql (Berulis et al. 1998), VX Sgr (Berulis et al. 1999; 
Pashchenko \& Rudnitskij 1999), RT Vir (Lekht et al. 1999), R Leo (Esipov et al. 1999), W Hya (Rudnitskij et al. 1999), U Ori (Rudnitskij et al. 2000), and RS Vir (Lekht et al. 2001). In this paper we now present a complete set of $113 \mathrm{H}_{2} \mathrm{O}$ line spectra obtained in 1981-2002 (see Figs. 1a-f).

$\mathrm{S}$ Per is within the region of the open cluster $\mathrm{h}, \chi$ Per (Per OB1 association) (Bidelman 1947; Humphreys 1970; 1975). The distance estimated from that of the cluster is $\approx 2.3 \mathrm{kpc}$. S Per is identified as an M-type supergiant, the most luminous member of the cluster $\left(L \approx 10^{5} L_{\odot}\right)$. Its mass is estimated to be $\approx 20 M_{\odot}(\mathrm{Gahm} \&$ Hultqvist 1976). According to different authors, the radius $R_{*}$ of S Per is within $(1.7-2.5) \times$ $10^{14} \mathrm{~cm}$ (Marvel 1996; Richards et al. 1999, and references therein).

In the 4th Edition of the General Catalogue of Variable Stars (Kholopov et al. 1987) S Per is classified as a semiregular $(\mathrm{SRb})$ variable. Its maximum amplitude in the visual is $7.9-12.0^{\mathrm{m}}$, while limits of the spectral type variations are M3Iae-M7, light cycle $822^{\mathrm{d}}$. In fact its light variations are much more complicated. Historic data shows the following: in 1920-1969 there were secondary waves with cycles of 300-700 and amplitude $\Delta m \approx 1^{\mathrm{m}}$ (Howarth 1976); in 1935-1961 $P_{1}=807-825^{\mathrm{d}}$ and $P_{2}=916-940^{\mathrm{d}}$ (Smith 1974; Leung \& Stothers 1977; Howarth 1978). Subsequent history of S Per was studied till 1990 by McConahay et al. (1989), Little-Marenin et al. (1991), and in 1983-1992 by Polyakova (2003), who also made parallel measurements of the intrinsic optical polarisation of S Per. Figure 2 a shows the visual light curve of S Per from 1980 to the present that is relevant to the interval before and during our $\mathrm{H}_{2} \mathrm{O}$ line monitoring of S Per.

In 1968-1974 the star was changing its visual brightness nearly periodically with $P \approx P_{\mathrm{GCVS}} \approx 822^{\mathrm{d}}$, and the amplitude was $\approx 2^{\mathrm{m}}$. Then at the beginning of 1975 there was a bright maximum to $\approx 8.2^{\mathrm{m}}$, followed by a deep minimum to $\approx 12^{\mathrm{m}}$. In July 1988 the star again had a bright maximum (JD 2447370 ) with a subsequent deep minimum (down to $>12^{\mathrm{m}}$ ). Till late 2000 it was oscillating with increased amplitude between $9^{\mathrm{m}}$ and 13 m . Thereafter, another period of "stagnation" began, similar to that of 1977-1986, but with a lower mean brightness, $\approx 10.5^{\mathrm{m}}$ (instead of $\approx 9.5^{\mathrm{m}}$ in 1977-1986). Thus, in S Per there are alternating intervals of large-amplitude Mira-like pulsations and of less regular variations of small-amplitude. In the past S Per also had intervals of strong light variations (1919-1927), followed by ones of smaller amplitude (1927-1938), and then again stronger changes, though at a lower mean level (see Bidelman 1947, and references therein), just like the curve for 1990-2002. In many respects the visual curve of S Per resembles that of VX Sgr, also with alternating large- and smallamplitude variations (Dinerstein 1973; Berulis et al. 1999).

Physical reasons for variability in red supergiants such as S Per have been discussed by many authors; see, e.g., Lovy et al. (1984), Li \& Gong (1994), Heger et al. (1997), Bono \& Panagia (2000), Guo \& Li (2002), and references therein. The conclusion is that the main cause of light variability in red supergiants is variation of the effective temperature due to stellar pulsation. In particular, Bono \& Panagia (2000) point out that with a transition to lower values of $T_{\text {eff }}$ the amplitude of brightness variations becomes larger, while the variations themselves become more chaotic. This is typical of late-type supergiants belonging to the SR variability type. Overall dimming of the star after a period of stronger oscillations may be due to subsequent enhanced mass-loss and ejection of a dust shell that screens the stellar radiation.

The star S Per is a known source of maser emission, first detected in the lines of $\mathrm{H}_{2} \mathrm{O}$ (Baudry \& Welch 1974), OH (Fillit et al. 1977; Bowers 1975), and $\mathrm{SiO}$ by Spencer et al. (1981).

In $\mathrm{S}$ Per thermal emission in the $J=2-1$ line of $\mathrm{CO}$ has been detected (González-Alfonso et al. 1998, Josselin et al. 1998). According to Josselin et al. (1998), the systemic LSR velocity of the star is $V_{*}=-37 \mathrm{~km} \mathrm{~s}^{-1}$, and the envelope expansion velocity is $17 \mathrm{~km} \mathrm{~s}^{-1}$.

Diamond et al. (1987) made another estimate of the velocity of S Per, determining it as a mid-point between the velocities of the two emission peaks in the $1612-\mathrm{MHz} \mathrm{OH}$ line, $-38.5 \mathrm{~km} \mathrm{~s}^{-1}$. In our discussion we will adopt the latter value of $V_{\mathrm{LSR}}$.

S Per has a high mass loss rate $\dot{M} \approx 2.7 \times 10^{-5} M_{\odot} \mathrm{yr}^{-1}$ (Gehrz \& Woolf 1971), estimated from IR data on the $10-\mu \mathrm{m}$ silicate feature), distinguishing it among other red supergiants. However, González-Alfonso et al. (1998) give a lower value of $\dot{M}$ based on their CO $J=2-1$ observations, $\approx 6.4 \times$ $10^{-6} M_{\odot} \mathrm{yr}^{-1}$.

The star S Per has been observed in the $22-\mathrm{GHz}$ $\mathrm{H}_{2} \mathrm{O}$ line on single antennae by Baudry \& Welch (1974), Dickinson (1976), Engels et al. (1988), McConahay et al. (1989), Barvainis \& Deguchi (1989), Comoretto et al. (1990), Little-Marenin et al. (1991), Palagi et al. (1993), Takaba et al. (1994), and Szymczak \& Le Squeren (1999). The profiles of the $\mathrm{H}_{2} \mathrm{O}$ line, obtained in these works at epochs close to our observations (when available), are in general consistent with our results.

$\mathrm{S}$ Per is also one of the stellar sources in which millimeter and submillimeter $\mathrm{H}_{2} \mathrm{O}$ maser lines have been detected: $3_{13}-2_{20}$ at $183 \mathrm{GHz}$ (González-Alfonso et al. 1998) and $5_{15}-4_{22}$ at $325 \mathrm{GHz}$ (Yates et al. 1995). The presence of these lines in the radio spectrum of $\mathrm{S}$ Per testifies to a high excitation of $\mathrm{H}_{2} \mathrm{O}$ molecules in its circumstellar envelope.

The 22- $\mathrm{GHz} \mathrm{H}_{2} \mathrm{O}$ maser in $\mathrm{S}$ Per has been mapped interferometrically by Diamond et al. (1987), Yates \& Cohen (1994), Marvel (1996), Richards \& Yates (1998), Richards et al. (1999, 2004), and Vlemmings et al. (2001, 2002). The $\mathrm{H}_{2} \mathrm{O}$ line maps obtained by these authors between October 1983 and December 1998 are generally consistent with each other. The $\mathrm{H}_{2} \mathrm{O}$ features on the maps trace an incomplete and asymmetric shell, extended in the NE-SW direction. Richards \& Yates (1998), and Richards et al. (1999, 2004) have come to conclusions about the strong coupling between stellar wind and dust, which directly affects also the $\mathrm{H}_{2} \mathrm{O}$ maser intensity. In their VLBA observations Vlemmings et al. (2001, 2002) measured circular polarisation of the $\mathrm{H}_{2} \mathrm{O}$ maser in $\mathrm{S}$ Per and found a magnetic field of up to $0.28 \mathrm{G}$ in the $\mathrm{H}_{2} \mathrm{O}$ masering region.

\section{Observations}

Our $\mathrm{H}_{2} \mathrm{O}$ observations were carried out on the 22-m radio telescope RT-22 of the Radio Astronomy Observatory 
(Astrospace Centre, Lebedev Institute of Physics, Russian Academy of Sciences) in Pushchino (Moscow Region). In 1981-1993 the receiving equipment included a 22-GHz maser amplifier, cooled by liquid helium, and the system noise temperature $T_{\mathrm{N}}$ was $200-300 \mathrm{~K}$, depending on the amplifier tuning and weather conditions. Since 1993 a cooled FET amplifier with $T_{\mathrm{N}}=80-120 \mathrm{~K}$ has been used. The receiver backend was a 128-channel filter-bank spectrometer, designed and made in the Sternberg Astronomical Institute with a frequency resolution of $7.5 \mathrm{kHz}\left(0.101 \mathrm{~km} \mathrm{~s}^{-1}\right.$ at the $\mathrm{H}_{2} \mathrm{O}$ line frequency). The frequency band required for analysis of the $\mathrm{H}_{2} \mathrm{O}$ spectrum of $\mathrm{S}$ Per, approximately $30 \mathrm{~km} \mathrm{~s}^{-1}$ wide in radial velocity, was covered by three contiguous tunings of the receiver by changing the frequency of the local oscillator. The integration time of each record was about $15 \mathrm{~min}$, yielding a $3 \sigma$ sensitivity of about $10 \mathrm{Jy}$.

The observing strategy in our $\mathrm{H}_{2} \mathrm{O}$ maser monitoring program is to obtain the spectra as frequently as possible. The interval between consecutive observations was usually from one to three months. As it depended mainly on the availability of the telescope time, the data are scarce in 1992-1995, though no drastic variations were obvious in this period. In recent years an important gap occurred between August 2000 and January 2001 because of the receiver malfunctioning. Since 2001 S Per has been monitored on a regular, nearly monthly basis.

Figures 1a-f present the entire set of the observed $\mathrm{H}_{2} \mathrm{O}$ line profiles of S Per. The vertical axis is the flux density $F_{v}$ in Janskys, while the horizontal axis is the radial velocity relative to the Local Standard of Rest $\left(V_{\mathrm{LSR}}, \mathrm{km} \mathrm{s}^{-1}\right)$. The maximum $V_{\mathrm{LSR}}$ range covered by our observations is from -53 to $-21 \mathrm{~km} \mathrm{~s}^{-1}$.

\section{Discussion}

A detailed study of the $\mathrm{H}_{2} \mathrm{O}$ maser variability in $\mathrm{S}$ Per was undertaken previously by Cox \& Parker (1978) in 1974-1977 (JD 2442 350-2 443 300). Their observations covered two light cycles of S Per, including its deep minimum near JD 2442840 , and they noted some correlation of the $\mathrm{H}_{2} \mathrm{O}$ data with visual brightness. In general, fluxes of individual features followed the optical variations, though with different phase delays.

Our observations of S Per cover a much longer time interval of more than 21 years. In Fig. 2 the integrated flux in the $\mathrm{H}_{2} \mathrm{O}$ line is plotted as a function of time. We divided the entire time interval of our observations into ten successive segments (labeled A to J), which can be identified with different activity periods of the $\mathrm{H}_{2} \mathrm{O}$ maser $\mathrm{S}$ Per.

Figure 3 shows the Fourier spectra for the visual light curve (a) and integrated $\mathrm{H}_{2} \mathrm{O}$-line flux curve (b). The FFT spectra were calculated for the time interval of 1987.0-2000.0, when the star was exhibiting pronounced light variations with a clearly expressed periodicity. Both spectra of Fig. 3 show a pronounced peak at a frequency of $\approx 0.0125 \mathrm{~d}^{-1}$, corresponding to a period of $800^{\mathrm{d}}$, which is fairly close to one of the periods found in S Per by Smith (1974) $\left(807-825^{\mathrm{d}}\right)$ and to the values of GCVS $\left(822^{\mathrm{d}}\right)$ and Polyakova (2003) $\left(850^{\mathrm{d}}\right)$. However, if extended to the entire interval of our $\mathrm{H}_{2} \mathrm{O}$ observations, the Fourier peaks are basically smeared out in view of the weak

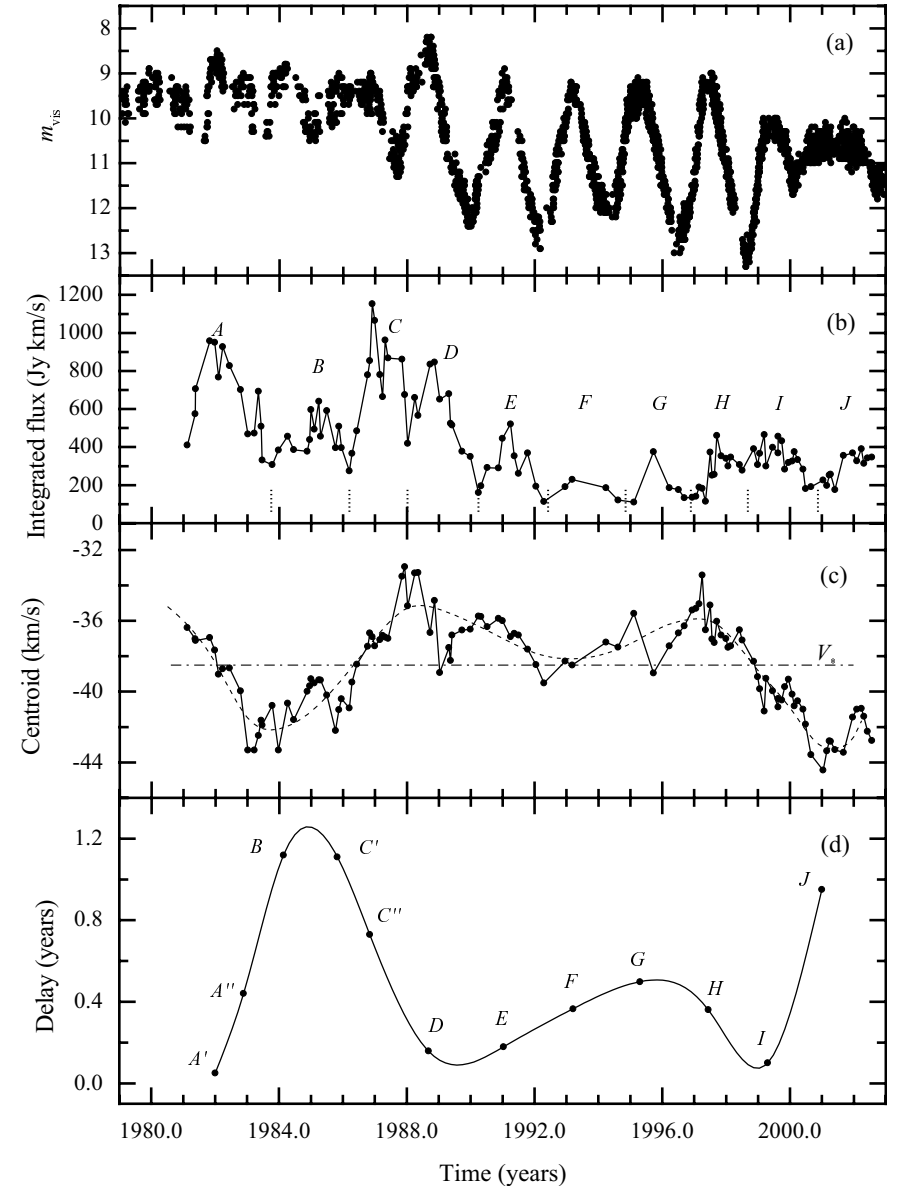

Fig. 2. Visual light curve of S Per, AFOEV data a), variations of the total flux b), velocity centroid c), delay (years) between the $\mathrm{H}_{2} \mathrm{O}$ line and visual maxima d). Vertical dotted lines divide the entire time interval into activity periods labeled $\mathrm{A}$ through $\mathrm{J}$; points $\mathrm{A}^{\prime} \mathrm{A}^{\prime \prime}$ and $\mathrm{C}^{\prime} \mathrm{C}^{\prime \prime}$ mark the limits of the integrated-flux events $\mathrm{A}$ and $\mathrm{C}$, respectively.

light variability of S Per outside the above-mentioned interval. Nevertheless, a more "latent" correlation between $m_{\text {vis }}$ is conserved for the entire interval of our $\mathrm{H}_{2} \mathrm{O}$ monitoring of $\mathrm{S}$ Per. As in our previous work on RS Vir (Lekht et al. 2001), we constructed a normalised cross-correlation function (Fig. 4) between the time dependence of $F\left(\mathrm{H}_{2} \mathrm{O}\right)$ and visual light curve:

$R(\tau)=\left\langle F_{\mathrm{H}_{2} \mathrm{O}}(t+\tau) F_{\mathrm{vis}}(t)\right\rangle /\left\langle F_{\mathrm{H}_{2} \mathrm{O}}(t+\tau) F_{\mathrm{vis}}(t)\right\rangle_{\max }$.

Here $\tau$ is the time delay between the light curve and maser variation. As the flux in the visual we used the value

$F_{\mathrm{vis}}=2.512^{14-m_{\mathrm{vis}}}$

in arbitrary units, referring to the light minimum magnitude of $14^{\mathrm{m}}$. We used 113 points of $F\left(\mathrm{H}_{2} \mathrm{O}\right)$ and 4300 points of $F_{\text {vis }}$ on an interval of $\tau=0-6250^{\mathrm{d}}(0-8 P)$. The correlation is obvious, as is the periodic character of the $\mathrm{H}_{2} \mathrm{O}$ line variations throughout the monitoring (1981-2002). A more detailed analysis of the curves of Figs. $2 \mathrm{a}$, b shows that the $\mathrm{H}_{2} \mathrm{O}$ flux variation has a delay with respect to the visual light curve; this delay is changing with time as well (Fig. 2d). The humps that are visible on curves of Figs. 2b, d probably mark some "superperiods" in the maser activity of S Per. An 11-year time span 


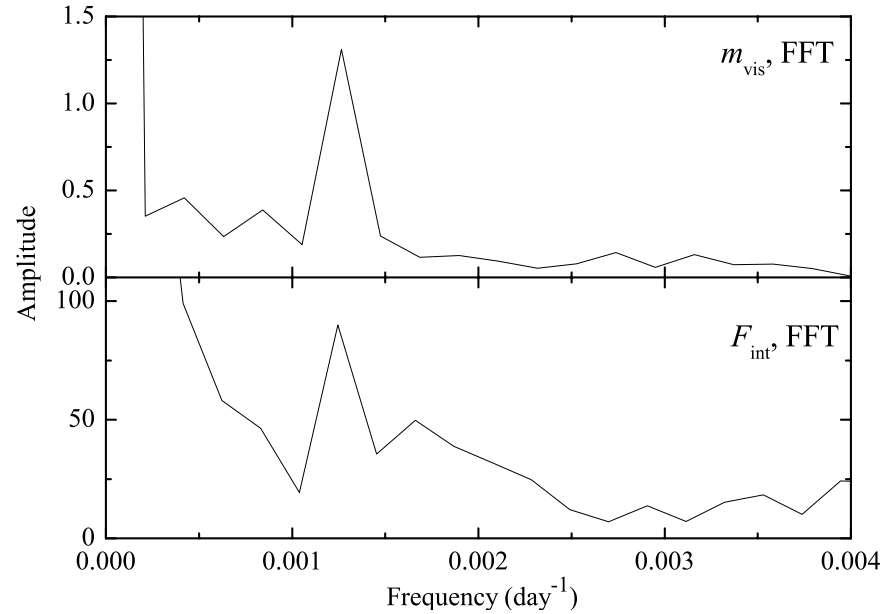

Fig. 3. Fourier spectra of the visual light curve and integrated flux curve (Fig. 2), calculated for the time interval 1987.0-2000.0. Both spectra show a peak at $f \approx 0.0125 \mathrm{~d}^{-1}$, corresponding to a period of $800^{\mathrm{d}}$.

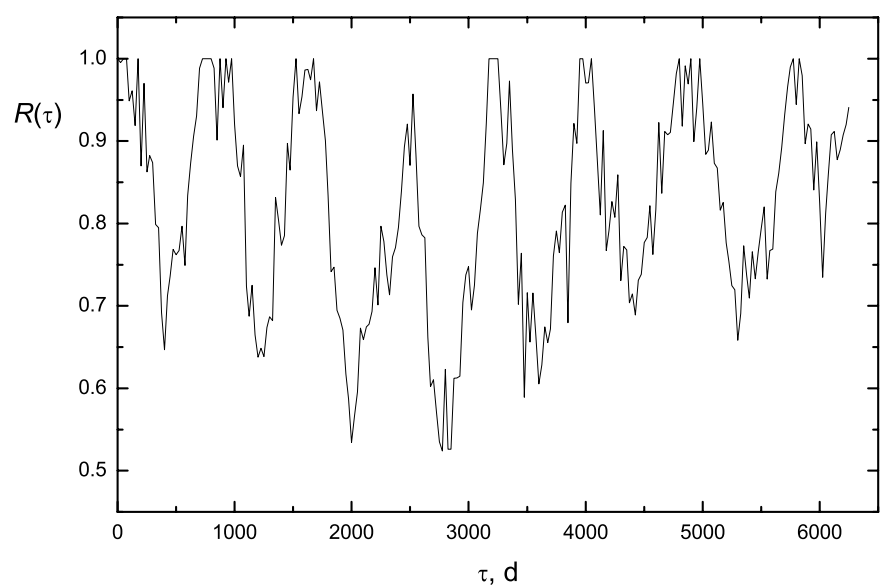

Fig. 4. Correlation function between the visual-range flux and integrated flux in the $\mathrm{H}_{2} \mathrm{O}$ line.

(BC-GH interval in Fig. 2d) and a 17-year one (BC-J) can be noted, though the time coverage of our monitoring is not yet sufficient to definitely confirm such long-term repeatability.

The $\mathrm{H}_{2} \mathrm{O}$ emission of $\mathrm{S}$ Per is subject to redistribution over the line profile. This is reflected in the variations of the $\mathrm{H}_{2} \mathrm{O}$ radial-velocity centroid (Fig. 2c), calculated as the weighted mean of the velocities of individual emission peaks

$V_{\mathrm{c}}=\frac{\sum_{i=1}^{n} F_{i} V_{i}}{\sum_{i=1}^{n} F_{i}}$,

where $F_{i}$ is the integrated flux of the $i$ th peak in the profile and $V_{i}$ is its radial velocity. The emission redistribution is visible when examining Fig. 5, which is an overlay of the spectra for separate segments and for the entire monitoring interval. Figure 6 presents the average spectra for the segments and the grand average.

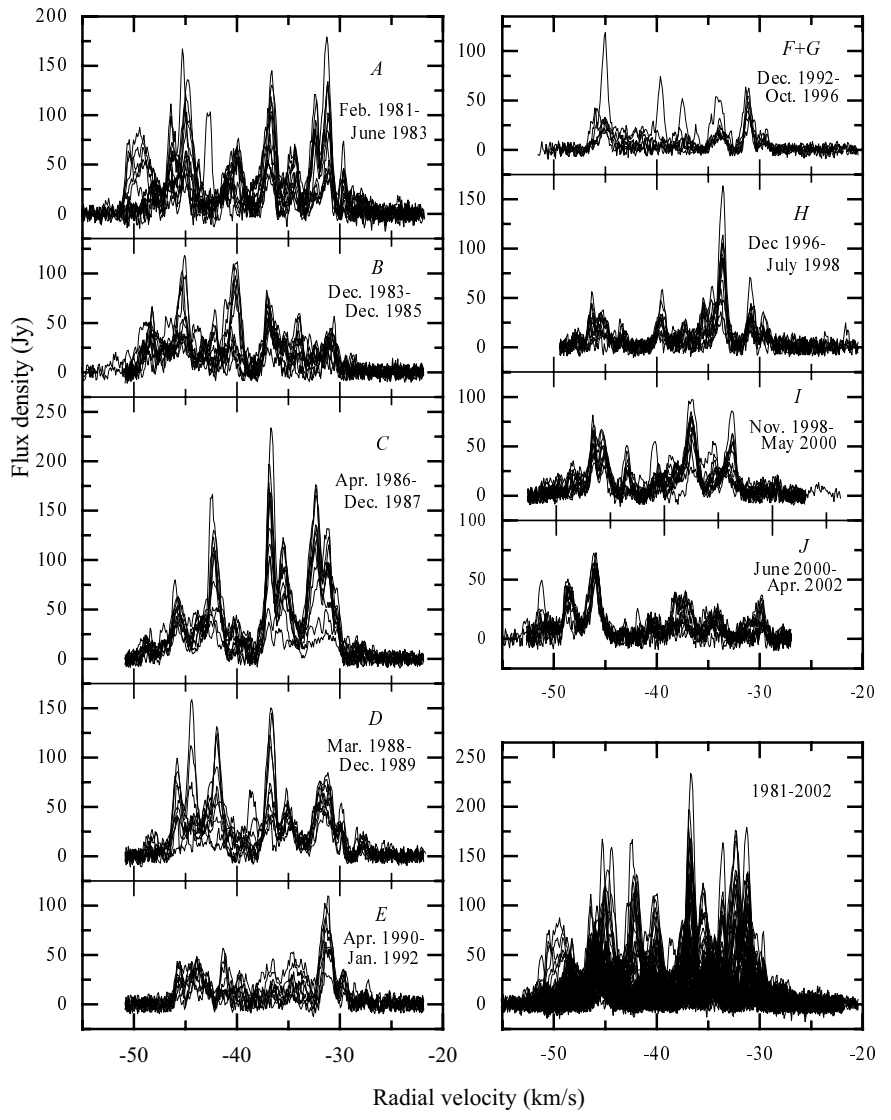

Fig. 5. Overlay of the spectra for each time period and for the entire time interval of our observations (1981-2002).

The main features of the $\mathrm{H}_{2} \mathrm{O}$ maser variations in $\mathrm{S}$ Per can be summarised as follows.

1. The total flux in the $\mathrm{H}_{2} \mathrm{O}$ line varies nearly periodically with a mean cycle of $\approx 800^{\mathrm{d}} \approx 2.1$ years (Fig. 3a, b), which is close to the cycles given by GCVS and Polyakova (2003) and to $P_{1}=807-825^{\mathrm{d}}$ of Smith (1974).

2. The strongest maxima are A, C, and D.

3. The activity minima fall on segments $F$ and $G$ (note, however, that our data are not complete here).

4. The visual brightness and $\mathrm{H}_{2} \mathrm{O}$ maser flux correlate.

5. The delay between the visual and maser maxima varies from one cycle to another within 0.01 to $0.5 P$ with a superperiod of 10-11 years (Fig. 2d). The presence of two secondary maxima in some activity cycles ( $\mathrm{A}$ and $\mathrm{C}$, segments labeled $\mathrm{A}^{\prime} \mathrm{A}^{\prime \prime}$ and $\mathrm{C}^{\prime} \mathrm{C}^{\prime \prime}$ in Fig. 2d) suggests the phase delay $\Delta \varphi=(n+(0.01$ to 0.5$)) P$ with $n=1($ not $n=3-5$ as in the $\mathrm{M}$-giant $\mathrm{H}_{2} \mathrm{O}$ maser $\mathrm{RS}$ Vir) - though the actual delay may be greater by more than a dozen stellar optical cycles, see discussion below.

6. The first maximum of the superperiod coincides with the minimum of the velocity centroid, while the second one does so with one of the centroid maxima (Fig. 2c). The interval with prevailing positive ("redshifted") velocities in 1988-1997 coincides with that of swinging optical variations of S Per (cf. Fig. 2a). 


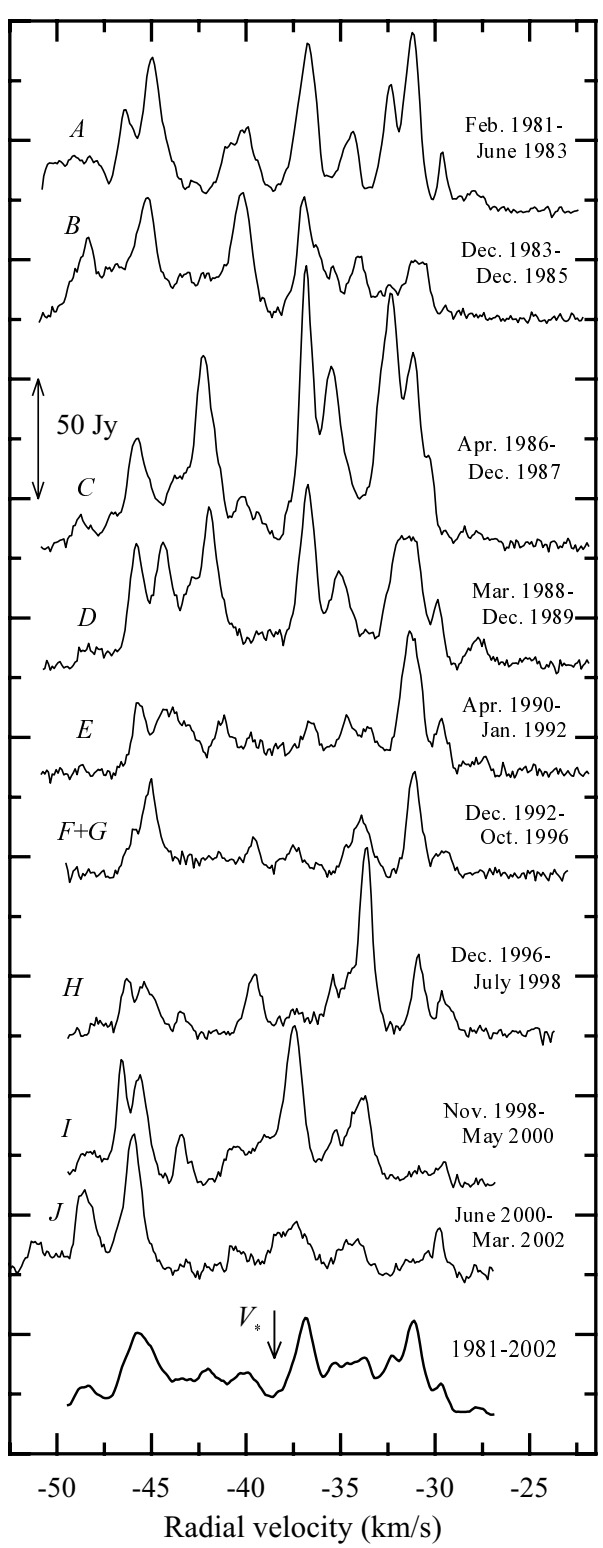

Fig. 6. Average spectra for each time period (A through $\mathrm{H}$ ). The grand average spectrum is shown in the bottom panel. Intervals $F$ and $G$ are joined together due to the small amount of data.

7. The mean spectrum, as well as the spectrum overlays infer a minimum at $V_{\mathrm{LSR}}=-38.5 \mathrm{~km} \mathrm{~s}^{-1}$, exactly at the spectrum centre and at stellar velocity $V_{*}$.

8. Only one spectral feature at $V_{\mathrm{LSR}}=-31.1 \mathrm{~km} \mathrm{~s}^{-1}$ persisted throughout our monitoring (Fig. 6).

9. The feature at $V_{\mathrm{LSR}}=-42.5 \mathrm{~km} \mathrm{~s}^{-1}$ was observed at intervals of enhanced maser activity ( $C$ and $D)$. In addition to this, it flared for a short time at the end of segment A. It appeared in only one spectrum. Hence we can conclude that it lasted no longer than 2.5 months (Fig. 6).

The generally accepted model for the $\mathrm{H}_{2} \mathrm{O}$ maser excitation is collisional pumping-see discussion in, e.g., Cooke \& Elitzur (1985), Field \& Gray (1988), Humphreys et al. (2001), and references therein. In particular, the recent Humphreys et al. model involves the excitation of $\mathrm{H}_{2} \mathrm{O}$ molecules by collisions with gas particles, mainly molecular hydrogen, as well as amplification of the $1.35-\mathrm{cm}$ background continuum. In its turn, this continuum is composed of the direct stellar radiation and emission from the surrounding dust. The model of Humphreys et al. (2001), elaborated for late-type giant stars (such as Mira variables), may also be valid for M-supergiants. This model reproduces the observed $\mathrm{H}_{2} \mathrm{O} 22-\mathrm{GHz}$ line profiles and VLBI maps well. It also accounts for the results of higher excitation $\mathrm{H}_{2} \mathrm{O}$ masers at 183,325 , and $321 \mathrm{GHz}$, the first two of which have been detected in S Per (González-Alfonso et al. 1998; Yates et al. 1995). The stellar continuum that is heating dust varies in time with changing effective temperature of the star (see the Introduction), which can account for the $\mathrm{H}_{2} \mathrm{O}$ maser variations - we discuss various aspects of this mechanism below.

As applied to the results of our monitoring, the model of collisional $\mathrm{H}_{2} \mathrm{O}$ pumping can explain the observed correlation between the visual brightness of the star and $\mathrm{H}_{2} \mathrm{O}$ maser emission flux (Figs. 3, 4) by several possible mechanisms: (1) periodic heating of circumstellar dust grains (and, as a consequence, of the surrounding gas) by variable IR emission of the star (Gómez Balboa \& Lépine 1986; Humphreys et al. 2001); (2) the effect of shock waves driven by stellar pulsation on the region of maser generation (Rudnitskij \& Chuprikov 1990; Berulis et al. 1998); (3) variations in density associated with changes in mass loss rate $\dot{M}$ (Elitzur 1992); (4) for the most blueshifted $\mathrm{H}_{2} \mathrm{O}$ maser features in the foreground of the stellar disc, a mechanism of amplification of stellar radio continuum at $\lambda=1.35 \mathrm{~cm}$ by pumped $\mathrm{H}_{2} \mathrm{O}$ molecules may be operating (Rudnitskij 1990).

Model (1) yields a small and almost time-constant phase delay $\Delta \varphi$ of the variation of integral line flux $F_{\text {int }}\left(\mathrm{H}_{2} \mathrm{O}\right)$ relative to the visual light curve. This is clearly not consistent with the observations. Moreover, a detailed analysis of Model (1) (Gómez Balboa \& Lépine 1986) shows that this model has some problems, because secondary infrared emission of the dust heated by stellar continuum instead has an antiinverting effect on $\mathrm{H}_{2} \mathrm{O}$, thus reducing the maser intensity.

In Model (2) the actual time delay can be $\Delta t=(n+\Delta \varphi) P$, i.e., could be increased by an integer number of the stellar light periods. Then, $\Delta t$ is the travel time of the shock, connected with the visual maximum of a star from the photosphere to the $\mathrm{H}_{2} \mathrm{O}$ maser layer in the circumstellar envelope. The time behaviour of $\Delta \varphi$ indicates that an increase in $F_{\text {int }}\left(\mathrm{H}_{2} \mathrm{O}\right)$ may be connected with one of the previous visual maxima, not with the latest one. A shock wave with velocity $v_{\mathrm{s}} \lesssim 10 \mathrm{~km} \mathrm{~s}^{-1}$ does not spend energy for ionisation of hydrogen and can propagate to rather large distances from the stellar surface, because its radiative losses are quite minor. The main energy losses of the shock are due to emission of heated dust behind the shock front and with dissociation and/or excitation of molecular hydrogen (if $\mathrm{H}_{2}$ is present in the circumstellar medium in a noticeable amount) (Rudnitskij 1997). The actual propagation time of the shock with $v_{\mathrm{s}} \approx 10 \mathrm{~km} \mathrm{~s}^{-1}$ from the stellar photosphere $\left(R_{\mathrm{ph}} \approx 10^{14} \mathrm{~cm}\right)$ to the $\mathrm{H}_{2} \mathrm{O}$ masering region $\left(R_{\mathrm{m}} \approx(1-2) \times 10^{15} \mathrm{~cm}\right)$ may be as long as 30 years, i.e., $\approx 14 P$. To reveal this effect of the central star variability on the maser shell, we should trace the correlation between $m_{\text {vis }}$ and 
$F_{\text {int }}\left(\mathrm{H}_{2} \mathrm{O}\right)$ on an interval that long and almost exceeding the time elapsed since the discovery of the $\mathrm{H}_{2} \mathrm{O}$ maser in $\mathrm{S}$ Per.

While not excluding the possibility of periodic shock impact as the cause of the $\mathrm{H}_{2} \mathrm{O}$ maser variations, Model (3) with short-term variations of mass-loss rate $\dot{M}$ (throughout the stellar light cycle $P$ ) may yield similar effects on the circumstellar gas density and, accordingly, on the maser pumping rate. Longer term variations of $\dot{M}$ also affect the radial position of the masering zone as $R_{\mathrm{c}}=\dot{M}^{2 / 3} V_{\text {exp }}^{-1}$, where $V_{\text {exp }}$ is the shell expansion velocity (Elitzur 1992). The masering zone moves in and out with changing $\dot{M}$. This readily accounts for the variations in time delay $\Delta \varphi$ between the light curves in the visual and in the $\mathrm{H}_{2} \mathrm{O}$ line. As shown by Kafatos \& Michalitsianos (1979), red supergiants may eject mass sporadically in episodes, which can also explain the $\mathrm{H}_{2} \mathrm{O}$ maser brightenings. In Model (3) the short-term mass-loss changes affect the entire maser zone simultaneously, so that the variations of the maser at the approaching, limb, and receding parts of the shell should be synchronous, as suggested by our Fig. 6 .

Finally, Model (4) predicts a nearly prompt response of the maser to stellar continuum, amplified by masering molecules (Rudnitskij 1990). This may be true for the most blueshifted features seen toward the centre of the $\mathrm{H}_{2} \mathrm{O}$ shell on maps of the $\mathrm{H}_{2} \mathrm{O}$ maser S Per (Richards et al. 1999, 2004). These features are at $V_{\mathrm{LSR}}=-44 \mathrm{~km} \mathrm{~s}^{-1}$-feature " $E$ " in McConahay et al. (1989) and Little-Marenin et al. (1991). If the effective temperature of the star has grown during its high optical maximum of 1987, the "radiophotosphere" (Reid \& Menten 1997) is also heated, thus increasing the radio continuum brightness temperature. In its turn, this radio continuum can be amplified by the $\mathrm{H}_{2} \mathrm{O}$ maser features foreground to the "radiophotosphere", which can be twice as extended as the optical. Indeed, this was the case during the high visual maximum of $S$ Per (1987) for the most negative-velocity features, which closely followed the optical brightness, as observed by us and by Little-Marenin et al. (1991). Compare, for instance, our $\mathrm{H}_{2} \mathrm{O}$ line profiles for May 5, 1988, and September 22, 1988 (Fig. 1c), where enhancement of the $-44-\mathrm{km} \mathrm{s}^{-1}$ feature (and other blueshifted features) between these two dates is obvious. Thus, Rudnitskij's (1990) model, too, can operate at least for these features.

Interesting changes can be traced in the $\mathrm{H}_{2} \mathrm{O}$ line structure of S Per. For instance, in 1981-1983 the $\mathrm{H}_{2} \mathrm{O}$ features at negative velocities (blueshifted) and at positive velocities (redshifted) with respect to $V_{*}=-38.5 \mathrm{~km} \mathrm{~s}^{-1}$ were approximately of equal strength (Figs. 5, 6). In 1983-1985 the blueshifted group was obviously stronger. In 1986-1987, again, redshifted features were brighter, and so on. Thus, the cycle of alternation is about two years, i.e., close to one of the fundamental periods of variability of S Per $\left(P_{1}=807-825^{\mathrm{d}}\right)$. In another M-type supergiant, VX Sgr, we observed a similar effect of redistribution of the $\mathrm{H}_{2} \mathrm{O}$ maser emission between the blue- and redshifted spectral features on a timescale of about ten years (Esipov et al. 1999; Pashchenko \& Rudnitskij 1999). We assumed the blueand redshifted features arise in lobes of a bipolar outflow, inferred by $\mathrm{H}_{2} \mathrm{O}$ VLBI maps, with the axis tilted to the line of sight at an angle of $\approx 60^{\circ}$ (Berulis et al. 1999).
A characteristic feature of S Per is its strong magnetic field. It was measured in observations of circular polarization of the $\mathrm{H}_{2} \mathrm{O}$ maser emission by Vlemmings et al. (2001, 2002), who obtained for $\mathrm{H}_{2} \mathrm{O}$ maser clumps the field strength of $B_{\|}=$ $279 \pm 30 \mathrm{mG}$ at a distance of a few hundred AU from the stellar surface. In our work on VX Sgr (Pashchenko \& Rudnitskij 1999), we attributed the above effect of the blue-red asymmetry variations for this star to redistribution of mass loss because of reversal of the general magnetic field of VX Sgr, measured by several authors in the $18-\mathrm{cm} \mathrm{OH}$ lines; see references in Pashchenko \& Rudnitskij (1999). However, in S Per the cycle of the blue-red exchange is considerably shorter. Furthermore, the bipolarity of the $\mathrm{H}_{2} \mathrm{O}$ shell of $\mathrm{S}$ Per, though elongated, is not prominent (Richards et al. 2004). Thus, the significance of a strong magnetic field of $\mathrm{S}$ Per for variations of its $\mathrm{H}_{2} \mathrm{O}$ maser is not yet clear.

\section{Conclusion}

In this work we have presented the profiles of the $\mathrm{H}_{2} \mathrm{O} 1.35-\mathrm{cm}$ emission line of the M-supergiant S Per for 1981-2002. Variability of the $\mathrm{H}_{2} \mathrm{O}$ maser $\mathrm{S}$ Per correlates with the visual light curve of the star. However, phase delay $\Delta \varphi$ of variations of $F_{\text {int }}\left(\mathrm{H}_{2} \mathrm{O}\right)$ with respect to the optical pulsations varied during our observations between 0.01 and $0.5 P$. If the variability of the $\mathrm{H}_{2} \mathrm{O}$ maser $\mathrm{S}$ Per is caused by periodic pumping action of pulsation-driven shock waves, the travel time of the shocks from the photosphere to $R_{\mathrm{c}}$, the inner boundary of the $\mathrm{H}_{2} \mathrm{O}$ maser region, may be as long as 14 stellar periods. Alternatively, maser variations may be connected with changing gas density and/or dust temperature in the masering region of the circumstellar envelope, caused by variations in mass-loss rate $\dot{M}$. In particular, long-term maser variations - the "superperiod" - also may be caused by changing $\dot{M}$. Furthermore, prompt correlation of the "blueshifted" feature at $-44 \mathrm{~km} \mathrm{~s}^{-1}$, located toward the star's disc, with visual brightness suggests amplification of the stellar radio continuum by masering $\mathrm{H}_{2} \mathrm{O}$ molecules (Rudnitskij 1990).

Acknowledgements. We thank the staff of the Pushchino Radio Astronomy Observatory for all their help with the $\mathrm{H}_{2} \mathrm{O}$ observations. The RT-22 telescope is supported by the Ministry of Industry, Science, and Technology of the Russian Federation, facility registration number 01-10. We are also grateful to Dr. Anita M. S. Richards for useful discussions and to the anonymous referee for helpful comments. This research made use of the AFOEV visual observations from the SIMBAD database, operated at Centre des données astronomiques de Strasbourg (France).

\section{References}

Barvainis, R., \& Deguchi, S. 1989, AJ, 97, 1089

Baudry, A., \& Welch, W. J. 1974, A\&A, 31, 471

Benson, P. J., Little-Marenin, I. R., Woods, T. C., et al. 1990, ApJS, 74, 911

Berulis, I. I., Lekht, E. E., Pashchenko, M. I., \& Rudnitskij, G. M. 1983, Sov. Astron., 27, 179

Berulis, I. I., Lekht, E. E., Munitsyn, V. A., \& Rudnitskij, G. M. 1998, Astron. Rep., 42, 346 
Berulis, I. I., Pashchenko, M. I., \& Rudnitskij, G. M. 1999, Astron. Astrophys. Trans., 18, 77

Bidelman, W. F. 1947, ApJ, 105, 492

Bono, G., \& Panagia, N. 2000, in The Impact of Large-Scale Surveys on Pulsating Star Research, ed. L. Szabados, \& D. Kurtz, ASP Conf. Ser., 203, 105

Bowers, P. F. 1975, AJ, 80, 512

Comoretto, G., Palagi, F., Cesaroni, R., et al. 1990, A\&AS, 84, 179

Cooke, B., \& Elitzur, M. 1985, ApJ, 295, 175

Cox, G. G., \& Parker, E. A. 1978, MNRAS, 183, 111

Diamond, P. J., Johnston, K. J., Chapman, J. M., et al. 1987, A\&A, 174, 95

Dickinson, D. F. 1976, ApJS, 30, 259

Dinerstein, H. 1973, JAAVSO, 2, 52

Elitzur, M. 1992, Astronomical Masers (Dordrecht: Kluwer), 265

Engels, D., Schmid-Burgk, J., \& Walmsley, C.M. 1988, A\&A, 191, 283

Esipov, V. F., Pashchenko, M. I., Rudnitskij, G. M., et al. 1999, in Asymptotic Giant Branch Stars, ed. T. Le Bertre, A. Lèbre, \& C. Waelkens (San Francisco: ASP), Proc. 191st Symp. IAU, 201

Field, D., \& Gray, M. D. 1988, MNRAS, 234, 353

Fillit, R., Proust, D., \& Lépine, J. R. D. 1977, A\&A, 58, 281

Gahm, G. F., \& Hultqvist, L. 1976, A\&A, 50, 153

Gehrz, R. D., \& Woolf, N. J. 1971, ApJ, 165, 285

Gómez Balboa, A. M., \& Lépine, J. R. D. 1986, A\&A, 159, 166

González-Alfonso, E., Cernicharo, J., Alcolea, J., \& Orlandi, M. A. 1998, A\&A, 334, 1016

Guo, J. H., \& Li, Y. 2002, ApJ, 565, 559

Heger, A, Jeannin, L., Langer, N., \& Baraffe, I. 1997, A\&A, 327, 224

Howarth, I. 1976, JBAA, 86, 210

Howarth, I. 1978, Light Curve 3, No. 1, 11

Humphreys, R. W. 1970, ApJ, 160, 1149

Humphreys, R. W. 1975, PASP, 87, 433

Humphreys, E. M. L., Yates, J. A., Gray, M. D., et al. 2001, A\&A, 379,501

Josselin, E., Loup, C., \& Omont, A. 1998, A\&AS, 129, 45

Kafatos, M., \& Michalitsianos, A. G. 1979, ApJ, 228, L115

Kholopov, P. N., Samus', N. N., Goranskii, V. P., et al. 1987, General Catalogue of Variable Stars, 4th ed. (Moscow: Nauka), vol. III, 30

Lekht, E. E., Mendoza-Torres, J. E., Pashchenko, M. I., \& Berulis, I. I. 1999, A\&A, 343, 241

Lekht, E. E., Mendoza-Torres, J. E., Rudnitskij, G. M., \& Tolmachev, A. M. 2001, A\&A, 376, 928

Leung, K.-C., \& Stothers, R. 1977, JBAA, 87, 264
Li, Y., \& Gong, Z. G. 1994, A\&A, 289, 449

Little-Marenin, I. R., Benson, P. J., McConahay, M. M., et al. 1991, A\&A, 249, 465

Lovy, D., Maeder, A., Noëls, A., \& Gabriel, M. 1984, A\&A, 133, 307

Marvel, K. B. 1996, The Circumstellar Environment of Evolved Stars as Revealed by Studies of Circumstellar Water Masers, Dissertation (Las Cruzes: New Mexico State University) http://www . aas.org/ marvel/POSTSCRIPT/DISS_NEW.ps

McConahay, M., Little-Marenin, I., \& Benson, P. J. 1989, JAAVSO, 18,107

Palagi, F., Cesaroni, R., Comoretto, G., et al. 1993, A\&AS, 101, 153

Pashchenko, M. I., \& Rudnitskij, G.M. 1999, Astron. Rep., 43, 311

Polyakova, T. A. 2003, Astrophys., 46, 465

Reid, M. J., \& Menten, K. M. 1997, ApJ, 476, 327

Richards, A. M. S., \& Yates, J. A. 1998, IrAJ, 25, 7

Richards, A. M. S., Yates, J. A., \& Cohen, R. J. 1999, MNRAS, 306, 954

Richards, A. M. S., Masheder, M. R. W., van Langevelde, H. J., et al. 2004, Proc. 7th European VLBI Network Symp. New Developments in VLBI Science and Technology, ed. R. Bachiller, F. Colomer, J. F. Desmurs, \& P. de Vicente (Toledo, Spain: Observatorio Astronómico Nacional), 209 http://www. oan.es/evn2004/WebPage/AMSRichards.pdf [arXiv: astro-ph/0501028]

Rudnitskij, G. M. 1990, in From Miras to Planetary Nebulae: Which Path for Stellar Evolution? ed. M.-O. Mennessier, \& A. Omont (Gif sur Yvette: Editions Frontières), 268

Rudnitskij, G. M. 1997, Ap\&SS, 251, 259

Rudnitskij, G. M., \& Chuprikov, A. A. 1990, Sov. Astron., 34, 147

Rudnitskij, G. M., Lekht, E. E., \& Berulis, I. I. 1999, Astron. Lett., 25, 467

Rudnitskij, G. M., Lekht, E. E., Mendoza-Torres, J. E., et al. 2000, A\&AS, 146, 385

Smith, H. A. 1974, JAAVSO, 3, 20

Spencer, J. H., Winnberg, A., Olnon, F. M., et al. 1981, AJ, 86, 392

Szymczak, M., \& Le Squeren, A. M. 1999, MNRAS, 304, 415

Takaba, H., Ukita, N., Miyaji, T., \& Miyoshi, M. 1994, PASJ, 46, 629

Vlemmings, W., Diamond, P. J., \& van Langevelde, H. J. 2001, A\&A, 375, L1

Vlemmings, W., Diamond, P. J., \& van Langevelde, H. J. 2002, A\&A, 394, 589

Yates, J. A., \& Cohen, R. J. 1994, MNRAS, 270, 958

Yates, J. A., Cohen, R. J., \& Hills, R. E. 1995, MNRAS, 273, 529 


\section{Online Material}


E. E. Lekht et al.: Variability of the $\mathrm{H}_{2} \mathrm{O}$ maser S Per, Online Material p 2

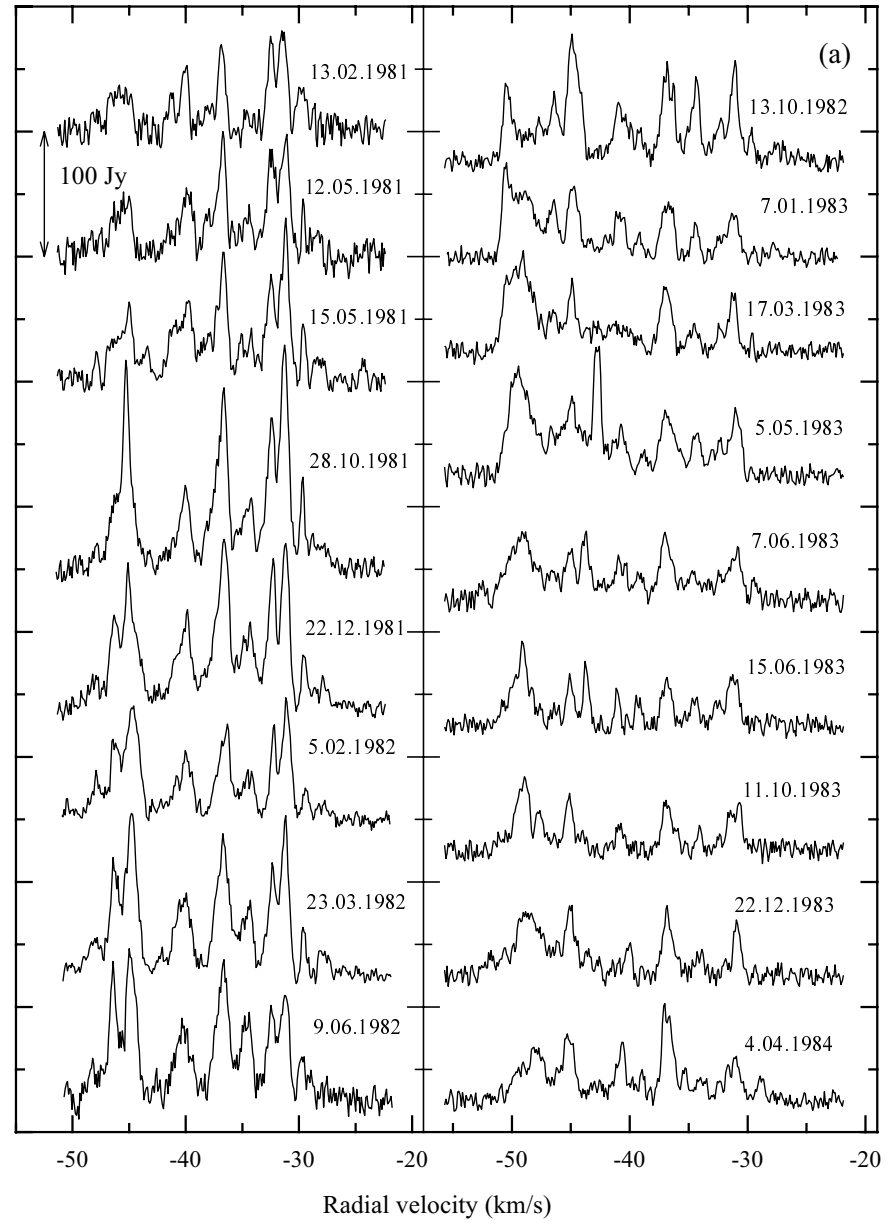

Fig. 1. Profiles of the $\mathrm{H}_{2} \mathrm{O}$ line emission of the star $\mathrm{S}$ Per. The vertical scale (flux density in Janskys) is common to all the graphs on the same panel. The horizontal axis is the radial velocity with respect to the Local Standard of Rest.

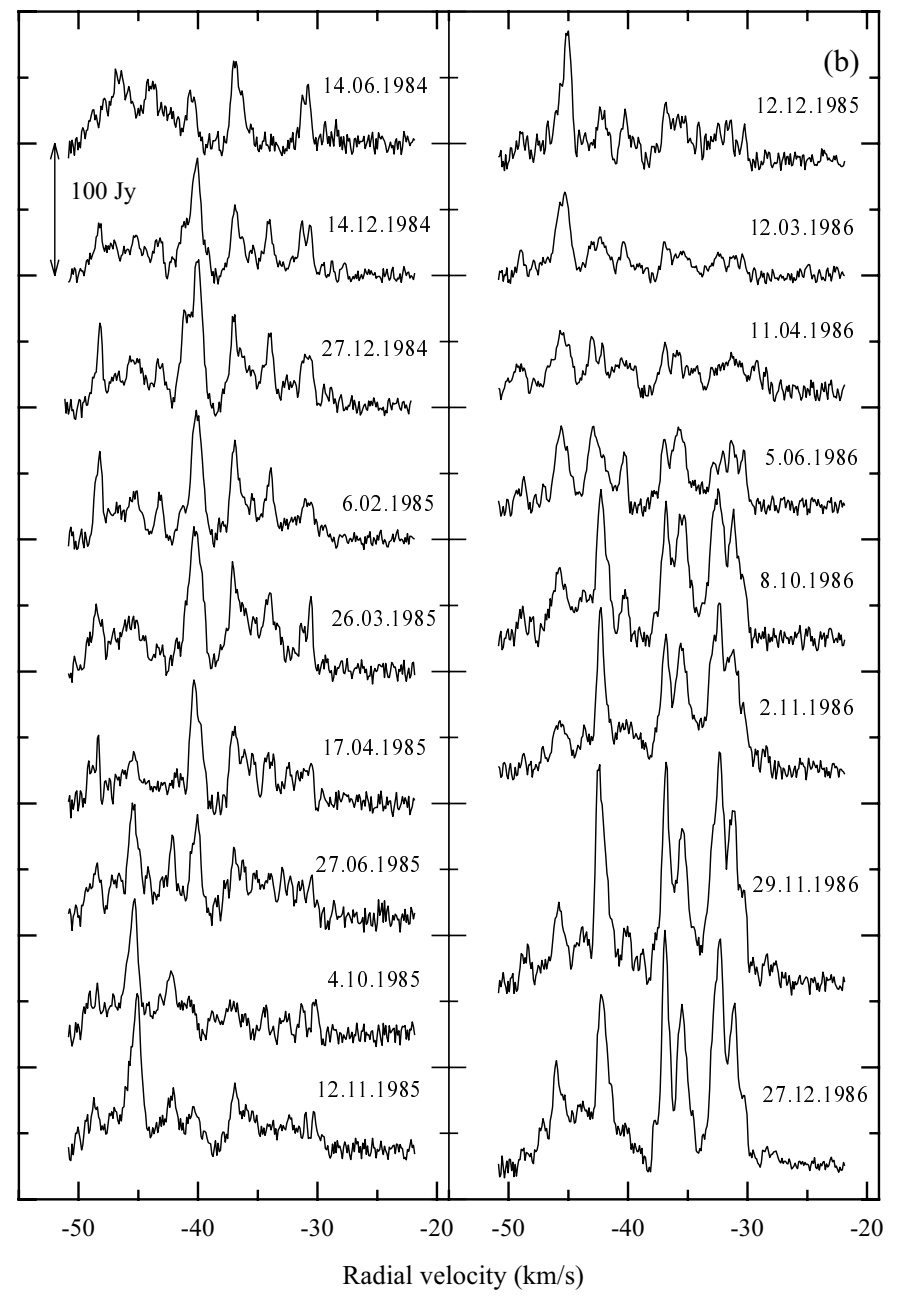

Fig. 1. continued. 
E. E. Lekht et al.: Variability of the $\mathrm{H}_{2} \mathrm{O}$ maser S Per, Online Material $p 3$

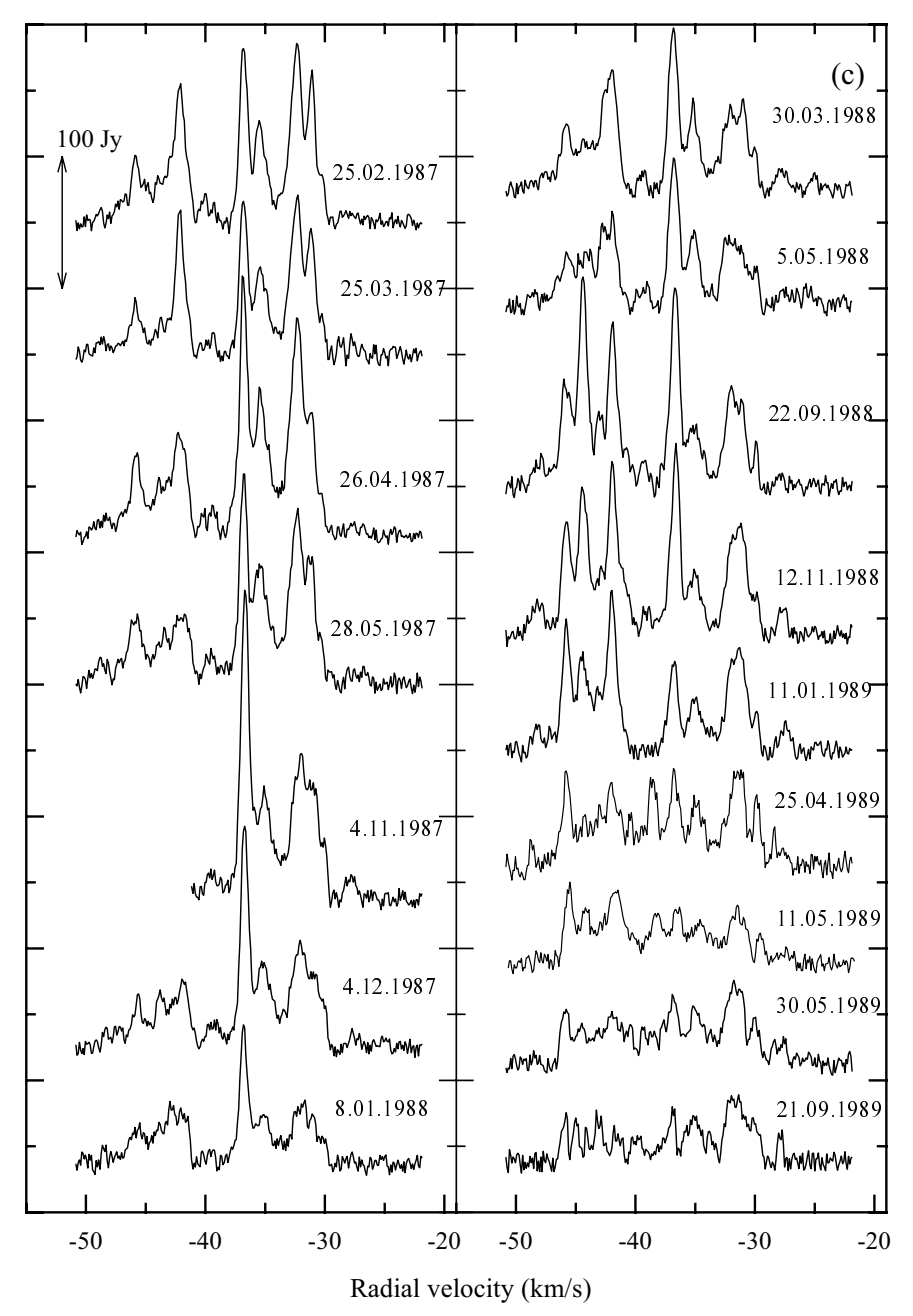

Fig. 1. continued.

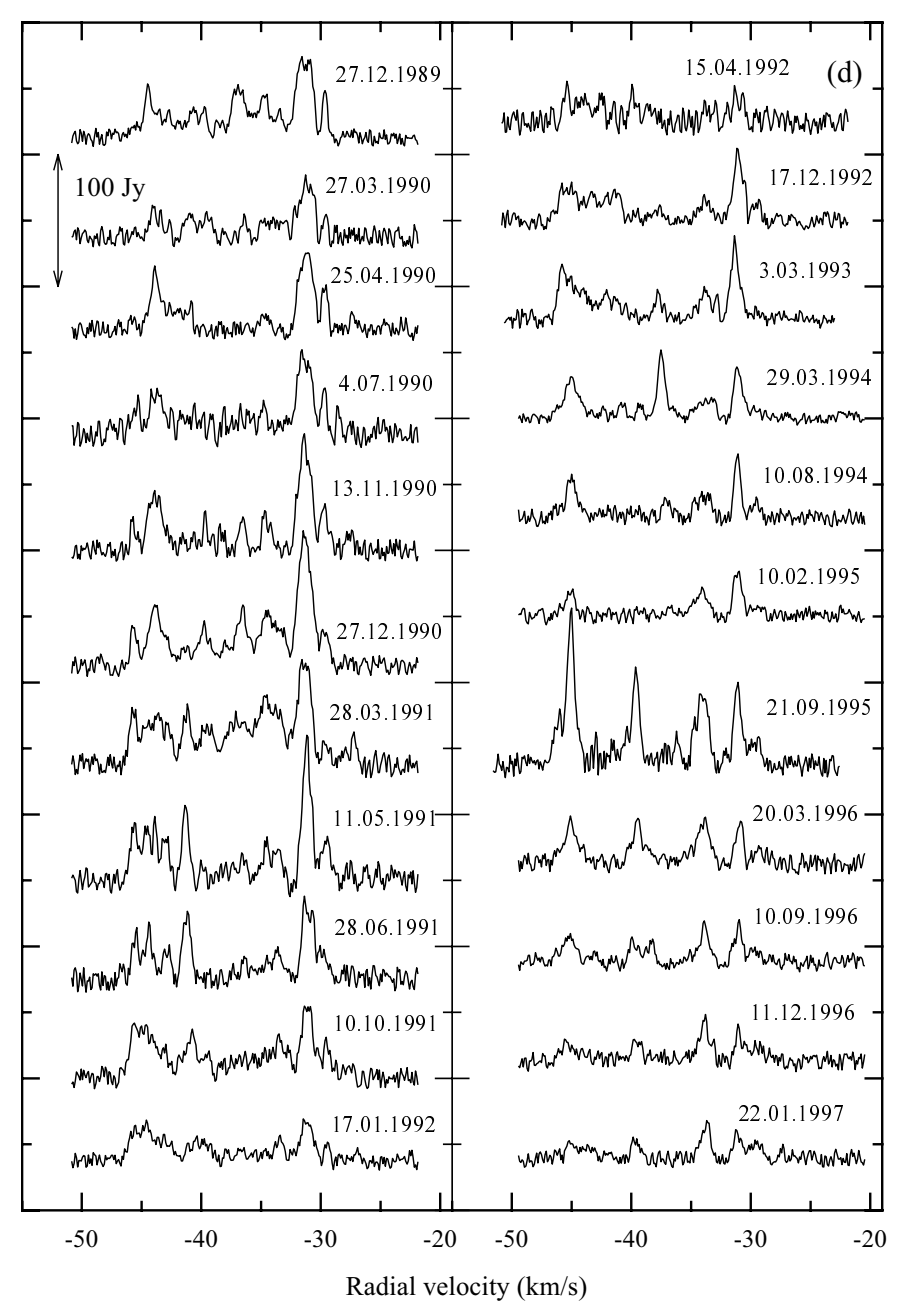

Fig. 1. continued. 
E. E. Lekht et al.: Variability of the $\mathrm{H}_{2} \mathrm{O}$ maser S Per, Online Material p 4

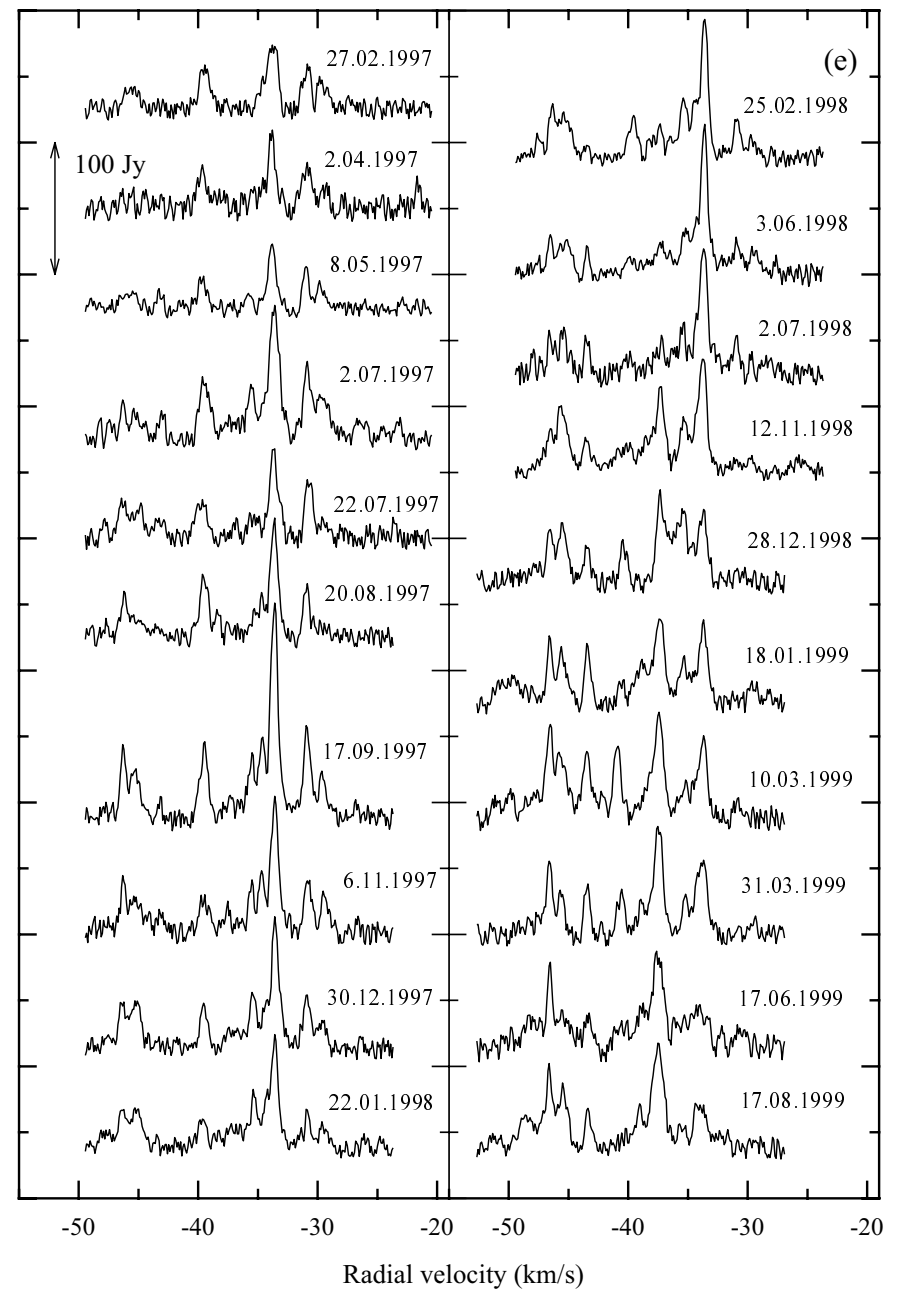

Fig. 1. continued.

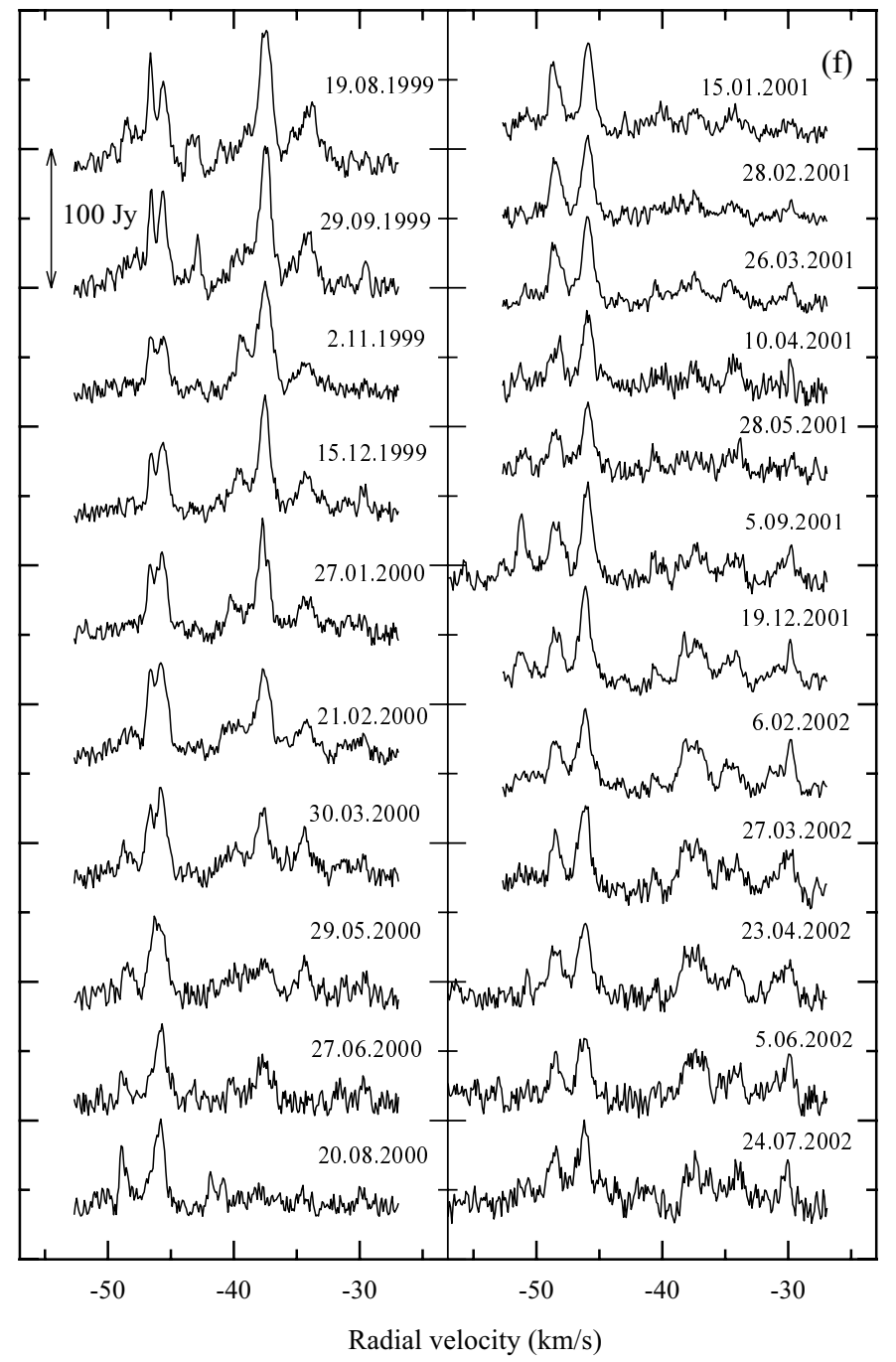

Fig. 1. continued. 